\title{
SCIENTIFIC KNOWLEDGE ACQUISITION DURING THE EXTENSION OF GSA: AN EXPERT SYSTEM FOR GENERIC SAFETY ANALYSIS
}

\author{
Mark R. Lehto \\ Department of Industrial Engineering, Purdue University, West Lafayette, IN 47907 (U.S.A.) \\ and James M. Miller \\ The University of Michigan, Department of Industrial and Operations Engineering, Ann Arbor, \\ MI 48105 (U.S.A.)
}

(Received January 19, 1987; accepted in revised form February 28, 1987)

\begin{abstract}
This paper describes an extension of GSA, a knowledge based expert system designed to help nonexperts perform generic safety analysis. GSA is implemented on the EMYCIN expert system shell, consists of approximately 150 safety related rules, and runs on a VAX $11-750$ computer. As originally implemented, GSA organized several generic safety problems within a well-structured tree and focused on problems within interface biomechanics. GSA also emphasized the application of theoretical principles instead of empirical data. To extend GSA, a specialized empirical knowledge base was developed that described the available slip resistance found for various combinations of surfaces, shoes, and con-
\end{abstract}

taminants. During knowledge acquisition, it became apparent that fundamental problems were present in much of the past research regarding to available slip resistance. We therefore chose to directly collect data during a large controlled experiment. The collected data was initially organized by applying statistical methods. After the statistical results were classified into conceptually meaningful categories, a set of rules was easily described and added to the system. The conclusion was that GSA describes an extendable framework that can organize and selectively apply very specialized safety ergonomics research.

\section{INTRODUCTION}

The size, diversity, and disorganization of the data in safety and ergonomics often impedes its application. Designers or other nonexperts in safety or ergonomics may find it difficult to isolate the small subset of relevant safety ergonomics data. More importantly, applying such data requires much synthesis of diverse principles, which is difficult even for safety practitioners highly familiar with safety (Johnson, 1980).

Directly providing relevant safety ergonomics knowledge during computer aided design is a possible solution to this problem. The need for sophisticated computer aided design methods has recently been been recognized by human factors experts (Askren, 1985; Coury, 1983). We propose that this need can often be satisfied by developing 
expert systems similar to the traditional models used to organize knowledge in safety and ergonomics.

This paper first addresses the use of traditional safety models to define a generic model that (1) organizes safety knowledge, and (2) is compatible with the knowledge representations used in expert systems. A description is given of GSA, an expert system equivalent to this model. An extension of GSA is then described, showing how detailed knowledge can be developed and added. An example illustrating use of the system is also given.

\section{BACKGROUND}

A subset of AI based computer programs, called expert systems, solves complex problems previously assumed to require human expertise (Stefik et al., 1982). Such programs emphasize the separation of a simple control structure from a highly structured knowledge base, thereby allowing the knowledge to be applied in flexible problem specific ways. For safety and/or ergonomics applications, such flexibility is attractive because a large knowledge base containing unorganized research findings and a few generic principles must somehow be distilled and applied to specific problems.

\section{Development of CAD / expert systems}

Expert systems require well-structured knowledge bases that are designed to be efficiently accessed, usually by simple search. Such knowledge bases partition complex problems into smaller subproblems and often are organized by hierarchies or other simple models that describe relationships between subproblems (Simon, 1969).

The development of well-structured knowledge bases is a labor-intensive process. Given that organized safety and ergonomics knowledge currently exists, even in partial form, much of this development work could be avoided (Lehto et al., 1984).

\section{Validation and maintenance of CAD / expert systems}

Validation and maintenance have been relatively neglected issues in expert systems research.
The techniques of systems analysis developed over years of information system design have not been adequately considered (Martins, 1984). Such neglect has resulted in costly systems that are difficult to maintain because of their complexity. The inherent difficulty of scientifically validating systems based on intuitive, nonstructured, so-called “expert knowledge", also poses a problem.

If expert systems in safety or ergonomics are carefully designed, both the maintenance and validation issues may become of less concern. Given that an underlying model cleanly partition the problems to be solved, it should be relatively easy to add new information to the system. In the most desirable case, the model would also organize carefully obtained "scientific" knowledge, making validation less difficult. Traditional methods of organizing safety and ergonomics knowledge can aid in attaining both objectives.

\section{Safety ergonomics knowledge structure}

Existing safety ergonomics knowledge is organized within accident models, safety standards, safety literature, accident statistics, and human experts. Importantly, both descriptive and empirical accident models correspond closely to knowledge structures commonly used by expert systems (Lehto, et al., 1984).

\section{Descriptive accident models}

Descriptive accident models organize safety knowledge. For example, the "energy countermeasures" model (Haddon, 1973) generically describes accident types, causes, and countermeasures. "Cause tree analysis" (Driessen, 1970) clarifies the relationship between multicausation and the accident sequence, and has been used to integrate high level principles found within the safety literature (Johnson, 1975). Other models describe important concepts such as sequential steps in decision-making (Ramsey, 1976; Surry, 1968), task analysis (Drury and Brill, 1983), and influences of personality factors (Taylor, 1976).

\section{Empirical accident models}

A smaller sub-set of accident models contain detailed knowledge applicable to specific safety problems. These models are empirically derived, and emphasize simple mathematical relations. Ex- 
amples are biomechanical (Chaffin et al., 1977), psycho-physiological (Mital, 1983), and material handling models (Coury, 1983).

\section{Combining descriptive and empirical models}

Both descriptive and empirical accident models structure safety ergonomics knowledge, but tend to either, (1) be so general that application to specific problems requires professional expertise, or (2) be so specific that many potential sources of knowledge and problem areas are completely neglected. Ideally, combining general and specific accident models within some larger knowledge structure would eliminate these problems.

The Management Oversight and Risk Tree (MORT) (Johnson, 1975; 1980) is an illustrative initial attempt at synthesizing safety principles in this way. There is a need for more effort along these lines because, in many areas of safety and ergonomics, both the knowledge and research is poorly organized (Lehto and Miller, 1986).

\section{THE STRUCTURE AND FUNCTION OF GSA}

GSA was not intended to immediately represent the most advanced empirical knowledge in safety and ergonomics. It was instead intended to be a skeletal framework to which new information could be incrementally added, eventually leading to a system that could be called expert in certain areas. The theory was that descriptive accident models could be used to define the gross structure of GSA and that this gross structure would both guide the application of specific principles and be easily extendable.

\section{The high-level knowledge base}

The knowledge base in GSA is defined by a model which has a meaningful form of abstraction at several different levels (Figs. 1-3). Each level corresponds to several subproblems which become more specific at lower levels. The production rules within the system are organized in accordance with this model. This results in a hierarchy of rules where general or "metalevel" rules define the overall problem solving strategy of the system, while more detailed rules develop specific conclu-

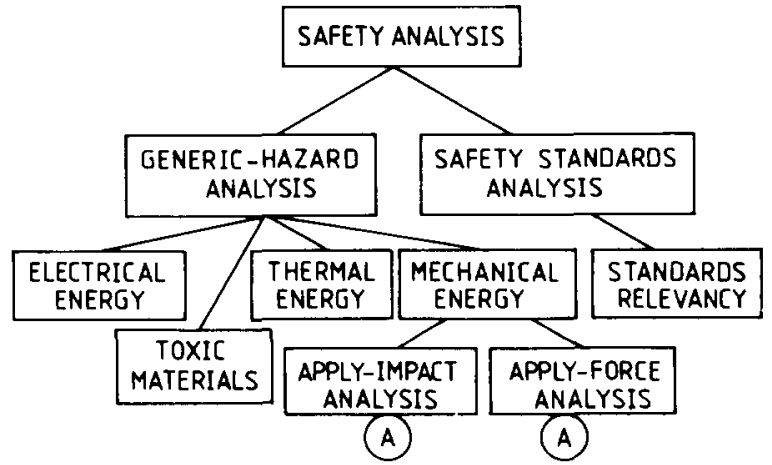

Fig. 1. The abstracted problem tree.

sions. Seventeen rules are used to specify this overall problem solving strategy.

At the top level (Fig. 1), two very general subproblems within safety analysis are distinguished. One is generic hazard analysis, the other is safety standards analysis. Within safety standards analysis, the immediate subproblem is to determine which standards are relevant. The system, as originally implemented, contained seven rules capable of selecting relevant standards for striking tools. Also defined, but not further analysed, were the subproblems pertaining to use related compliance, materials compliance, tested compliance, and manufacturing compliance.

Within general hazard analysis, one subproblem is to èvaluate the hazards associated with hazardous/toxic materials. Other obvious subproblems are described by Haddon's (1973) energy model, which distinguishes between the hazards associated with electrical, thermal, and

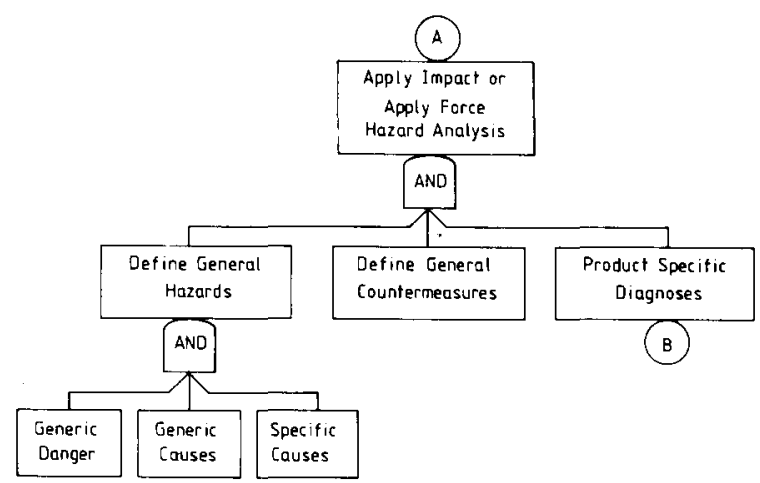

Fig. 2. A high level description of apply force or apply impact hazard analysis. 


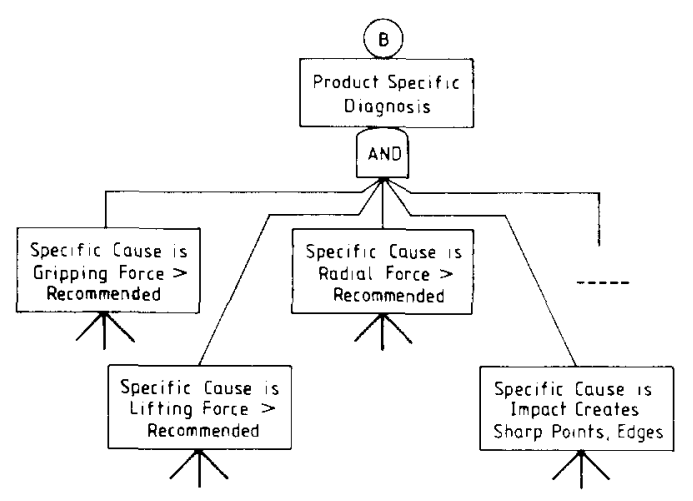

Fig. 3. The relation of specific danger causes to the product specific diagnosis.

mechanical energy transfer. Each subproblem has an associated form of generic hazard analysis which again can be described with a set of subproblems. RULE006 in Table 1 shows how the subproblems at this level are organized by a single rule.

\section{The focused knowledge base}

The focus in GSA in on safety problems within interface biomechanics, a subset of those involving the transfer of mechanical energy. Interface biomechanics is specifically concerned with the mechanical interface between the human and physical objects. Example tasks involving interface biomechanics include walking, lifting, carrying, gripping, striking, and so on.

Safety problems in interface biomechanics can be separated into those which involve impact and/or the application of force. Figure 2 illustrates the general subproblems described by GSA that fall within impact and/or force related hazard analysis. These problems and the sequence they are performed in are: (1) define general hazards, (2) define general countermeasures, and (3) develop a product specific diagnosis. Thirtythree rules are used to determine the general hazards and countermeasures. Seventy rules are used to develop and display product specific diagnoses.

\section{General hazard definition}

General hazards are defined in GSA by first determining the generic danger, then the generic causes of the generic danger, and finally the
TABLE 1

Two example rules used in GSA

RULE006

If: (1) Mechanical-energy-related-hazards-analysed,

(2) Electrical-energy-related-hazards-analysed,

(3) Thermal-energy-related-hazards-analysed, and

(4) Toxic-material-related-hazards-analysed

Then: (1) Generic-hazards-analysed = yes (1.0), and

(2) Display generic-hazards-analysed

RULE029

If: (1) Force-applied-by-human-when-applying-impact is traced,

(2) Human-arm-length is traced, and

(3) Inertia-at-shoulder is traced

Then: Angular-acceleration-at-product-head $=$ force-appliedby-human-when-applying-impact * human-arm-length / inertia-at-shoulder

specific causes of the generic danger. The hazard definition within GSA becomes progressively more detailed as this sequence of activity nears completion.

To describe the generic danger, the first subproblem is to selectivity assign to the analysed product the basic properties of striking, prying, or portability. These properties reflect the initial emphasis on developing a knowledge base for handtools. More importantly, they correspond to basic product functions which can be easily related to generic dangers, the second subproblem. For example, when analysing striking tools, a generic danger is "the transfer of kinetic energy to the human". For prying tools a generic danger is "the buildup and rapid transfer of potential energy to the human". For portable products a generic danger is "an excessive or stressful application of force by the human", as in lifting.

For each generic danger, there is a generic cause that can be further broken down into specific causes. For example, if the generic danger is "the transfer of kinetic energy to the human", a generic cause is "the breakage of important connections within or between the human and product". Several more specific causes then define which important connections can be broken in terms of task and product specific leverage, radial, tangential, and connect forces. The connections are described by the possible interfaces between and within the human and product (hand to handle, 
foot to ground, prying surface to target object, and so on). The forces are described by the basic product function. For example, striking will involve radial, tangential, and connecting forces and prying will involve leverage and connecting forces.

Seventeen rules are used to describe this general relationship between generic danger, generic causes, and specific causes, as function of basic product functions.

\section{General countermeasure definition}

Each general countermeasure is the inverse of particular generic and specific causes. Countermeasures to the danger caused by broken connections include increasing the connection forces and reducing the radial, tangential and leverage forces. Countermeasures to force application related stress are to reduce the required force and to increase the acceptable force. Countermeasures to contact with sharp points or edges include designing out sharp points and edges, designing guards which cannot be removed, using materials which do not form sharp points or edges after shattering, and so on.

Sixteen rules described the relationship between general countermeasures and causes.

\section{Product specific diagnoses}

The specific causes of danger described above are hypothetical problems for an analysed product, because they are entirely developed by applying general principles. The product specific diagnosis therefore involves the analysis of each specific cause found to be relevant during the generic analysis (Fig. 3). Certain specific causes are relevant for more than one product function (gripping force greater than recommended), others are unique (impact creates sharp points and edges).

The analysis mainly consists of estimating and then comparing the forces described within each relevant specific cause, for each applicable product function. If a disconnection force is found to exceed the opposing connection force, the specific cause is no longer hypothetical and a safety problem has been uncovered for the product. Exerted forces are also compared to allowable limits. For example, one very general limit is a $50 \mathrm{lb}$ lifting force when the product is portable and the subject is a male (based on Snook (1978) and the implication that portable products will occasionally be moved-other more specific limits based on NIOSH (1980) are also derivable).

Connecting and disconnecting forces are calculated using values of product-, user-, and environment-specific factors that are obtained from the system's user. At this level, the knowledge base is consequently organized in terms of the parameters needed to calculate particular forces. For example, in order to calculate the gripping force required during a prying task, coefficients of friction, lever arms, and applied forces must be determined. Determining values for each of these parameters requires that other parameters be determined, and so on.

The majority of rules relate such parameters to the hypothetical causes of safety problems (i.e. RULE029 in Table 1). Table 2 organizes parameters used in the initial version of GSA. Unfortunately, space constraints make it impossible to provide a detailed description.

\section{THE INITIAL IMPLEMENTATION OF GSA}

The GSA initially consisted of a knowledge base of 127 rules and was implemented on a VAX 11-750 computer using the EMYCIN computer program (Van Melle, 1980). The system performed generic safety analysis in the area of interface biomechanics and focused on handtool safety (Lehto, 1985). It also had a very general, but essentially undeveloped capability to perform generic safety analysis in other areas (Fig. 1).

Certain characteristics of the resultant system are particularly interesting. Two such characteristics discussed below include, (1) meaningful levels of abstraction, and (2) sophisticated user-system interaction. A session with the extended version of GSA is discussed later (Table 10).

\section{Meaningful levels of abstraction}

GSA completes a generic analysis, before attempting a product specific diagnosis. GSA is unique, when compared to other AI applications, because the solutions obtained at each level are meaningful and of value. Table 3 summarizes selected generic outputs obtained during the analysis of a clawhammer. Note that the generic outputs are similar to the points given in general 
TABLE 2

Parameters considered in product specific diagnoses

\begin{tabular}{|c|c|}
\hline Apply impact related & Apply force related \\
\hline 1. Gripping force analysis & 1. Gripping force analysis \\
\hline a. Effects of product & a. Effects of product \\
\hline 1. product weight & 1. product weight \\
\hline 2. handle length & 2. handle length \\
\hline 3. handle material & 3. handle material \\
\hline 4. handle COF & 4. handle COF \\
\hline b. Effects of environment & b. Effects of environment \\
\hline 1. handle contaminents & 1. handle contaminents \\
\hline c. Effects of user & c. Effects of user \\
\hline 1. Expected grip strength & 1. Expected grip strength \\
\hline 2. Skin COF & 2. Skin COF \\
\hline 2. Lifting force analysis & 2. Lifting force analysis \\
\hline a. Effects of product & a. Effects of product \\
\hline 1. product weight & 1. product weight \\
\hline 2. product dimensions & 2. product dimensions \\
\hline 3. handle dimensions & 3. handle dimensions \\
\hline b. Effect of user & b. Effects of user \\
\hline 1. sex & 1. $\operatorname{sex}$ \\
\hline 3. Flying objects analysis & 3. Push/pull force analysis \\
\hline a. Effects of product & (Prying task) \\
\hline 1. product weight & a. Effects of product \\
\hline 2. handle length & 1. product weight \\
\hline 3. handle COF & 2. handle length \\
\hline 4. head material & 3. handle COF \\
\hline 5. heat/handle connection strength & 4. head material \\
\hline 6. head hardness & 5. head/handle connection strength \\
\hline 7. handle shear strength & 6. prying surface strength \\
\hline b. Effects of environment & 7. handle shear strength \\
\hline 1. target object hardness & b. Effects of environment \\
\hline c. Effects of task & 1. target-earth connection strength \\
\hline 1. forces applied & c. Effects of task \\
\hline 2. angular and linear velocities & 1. forces applied \\
\hline 3. radial disconnect force between head and handle & 2. resultant leverage \\
\hline d. User effects & 3. tangential shear force between head and handle \\
\hline 1. weight of arm links & d. User effects \\
\hline 2. grip strength & 1. grip strength \\
\hline 3. force applied & 2. force applied \\
\hline
\end{tabular}

safety texts, and might remind a designer of obvious, but easily forgotten principles.

Table 4 presents part of the diagnosis for a particular claw-hammer. During the dialogue (user responses follow double asterisks), knowledge regarding the effects of a common environmental contaminant (water) is considered by GSA when calculating a coefficient of friction (COF) between the hammer's handle and the human's hand. Given this COF value and other task related information, GSA determines that a potential hazard is present. Specifically, GSA concludes that "it is foreseeable that a larger than recommended $40 \mathrm{lb}$ gripping force will be required while applying impact with this particular hammer". The system then moves on to evaluate other diagnoses.

\section{Sophisticated user-system interaction}

Many aspects of GSA are likely to aid user-system interaction. These include, (1) sophisticated explanation facilities, (2) structured query dialogue, (3) multiple lines of reasoning, and (4) tolerance of user uncertainty. 
TABLE 3

Selected generic outputs obtained during safety analysis of a claw hammer

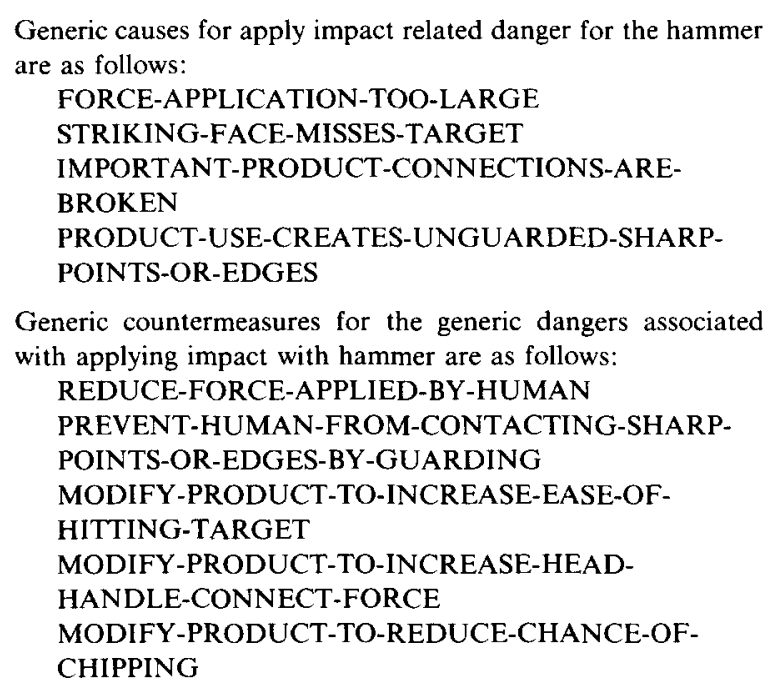

\section{Sophisticated explanation facilities}

One of the more interesting facilities of GSA is its ability to explain "why" and "how" certain conclusions are made. Such outputs are a direct consequence of the model underlying GSA and

\section{TABLE 4}

Outputs obtained during the diagnosis of a special safety problem for a claw hammer

(15) Is WOOD the material from which the hammer's handle is composed?

** YES

(16) Is OIL one of the foreseeable materials in the environment the hammer is used within?

** NO

(17) Is WATER one of the foreseeable materials in the environment the hammer is used within?

** YES

The coefficient of friction between hand and hammer's handle is as follows: 0.25

A specific safety problem for the hammer is as follows:

THE-FORESEEABLE-REQUIRED-GRIP-FORCE-FOR-

THE-PRODUCT-IS-LARGER-THAN-RECOMMENDED

The foreseeable required gripping force exerted by the human to keep the hammer from slipping from the human's hand is as follows:

40.007 POUNDS
TABLE 5

Example application of the WHY command

(17) What is the Rockwell-C hardness value of the hammer's face?

** WHY

[i.e. WHY is it important to determine the Rockwell hardness of the PRODUCT-2 face?]

[1.0] This will aid in determining special apply-impact related safety problems for PRODUCT-2.

It has already been established that

[1.1] apply-impact is one of the generic designed functions of the hammer, and

[1.2] tangential-force-gt-connect-force is one of the specific reasons important product connections may be broken for the hammer during impact application

Therefore, if

[1.3] the Rockwell hardness of the hammer head is less than or equal to foreseeable hardness value on the Rockwell $\mathrm{C}$ scale of target objects then

(1) It is definite (1.0) that hitting-hard-objectswith-hammer-will-cause-flying-chips is one of the special apply-impact related safety problems for the hammer.

Author: MRL

[RULE033]

the ability of the EMYCIN program to utilize this structure to create meaningful explanations. Of interest is that these explanations are given in English rather than cryptic code.

These abilities are partially demonstrated in Table 5. For that exchange the user's query of why GSA requires information concerning the Rockwell hardness of the hammer's head is answered by stating "this will aid in determining special apply-impact related safety problems". GSA then states what it already knows to be true about the particular problem and explains that if the Rockwell hardness of the hammer's head is less than the Rockwell hardness of the struck object, flying chips are a special apply-impact related safety problem for the hammer.

Another generic query allows the user to ask how GSA developed certain conclusions. Referring back to the previous example, if the user asks how it was determined that apply-impact was a designed product function, GSA explains "a designed function is apply-impact because the product's name is hammer". To an additional how 
TABLE 6

An example expanded query given by GSA

(12) Do you wish to input an estimate of the force the human exerts upon the handle of the hammer when applying impact? If you are unsure type return information?

** RETURN INFORMATION

This force estimate is used by the program to estimate the maximum linear velocity of the head of the hammer. If a value is not given, the program will not be able to calculate this velocity and will instead ask you for an estimated maximum linear velocity.

query, GSA answers "you said the product's name is hammer".

GSA is also able to provide extensive background information regarding certain questions it asks of the user. Table 6 documents such an exchange. In another similar example, GSA explains to the user that the weight and length of a human's arm are needed in order to calculate the forces exerted while applying impact with a handtool and also explains its ability to provide default values.

\section{Structured query dialogue}

GSA queries the user for particular facts when they are needed to solve a specific subproblem. Once GSA can tell from a user answer that a particular reasoning chain is a dead end, or that the problem is solved, it backs up to a higher level subproblem. The questions follow a logical pattern, because the reasoning chains are explicitly defined by the underlying model which organizes the subproblems.

\section{Multiple lines of reasoning}

Often GSA is able to reach a conclusion in more than one way. For example, when calculating the tangential forces exerted while applying impact, the system can obtain the same answer from either an estimate of the applied force or the maximum angular velocity. GSA can directly ask the user for the values of such parameters and also has the ability to estimate values if the user is unable or unwilling to provide them. The earlier example (Table 4) illustrates this flexibility, as the user could have simply given GSA the value for COF.

\section{Tolerance of user uncertainty}

GSA is also able to accept user responses of arbitrary certainty because of the built in capabili- ties of EMYCIN (Van Melle, 1980). Any response by the user can have an associated certainty factor ranging from -1 to 1 . A minus one indicates the user is sure the response is untrue while a one indicates the user is sure the response is true. A zero, on the other hand, indicates complete uncertainty. The uncertainty in responses is propagated throughout the developed conclusions by EMYCIN (see Shortliffe and Buchanan, 1984).

Returning to the example given in Table 4 , the user could have included a certainty value of 0.5 with the answer WOOD to the question regarding the handle material. This would cause GSA to also ask if STEEL or RUBBER was the handle material. Affirmative answers, with certainty of 0.5 , to these latter questions would result in the calculation of three COF values, each with a certainty of 0.5 . An affirmative answer, with a certainty of 1 for any of these materials would result in a single COF estimate with certainty of 1 .

\section{EXTENSION OF THE GSA}

Several significant safety problems within interface biomechanics were not originally considered in the GSA. Among these problems, slips and falls posed an interesting opportunity to extend the system. Of interest was that empirical knowledge is especially applicable during the analysis of slips and falls. The area also was sufficiently different from handtool safety to allow maintenance issues to be evaluated.

\section{Scope of the extension}

One indicator of the potential of slips and falls is the available surface slip resistance, traditionally expressed as coefficient of friction (COF). In spite of the numerous studies that have evaluated 
surface slip resistance, little detailed information is available in a form easily applied by designers. This led us to conclude that an extended version of GSA would be of value to designers, given that it could accurately predict the available slip resistance in a variety of circumstances. We therefore addressed the potential of extending GSA so as to have specialized expert ability in this problem area.

\section{Knowledge acquisition}

It is well-known that the available surface slip resistance is infuenced by flooring surface materials and textures, shoe soles, and contaminants. Predicting the available slip coefficient as a function of these qualitative factors is actually a classification problem; a category of problems for which rule based expert systems are especially appropriate.

As a first step in knowledge acquisition, an initial literature survey immediately revealed the difficulty of predicting surface slip resistance on the basis of the published literature. Fundamental problems were present, such as questionable testing methods and past failures to factorially investigate the influence of variables. Because of these problems, we chose to collect data in a controlled experiment. This moderately reduced the scope of the problem and increased our confidence in the validity of the data upon which the system would depend.

\section{Data collection}

A complete factorial experiment was performed (for details see Miller, Lehto, and Rhodes, 1987) using a testing procedure similar to that recom- mended in Federal Specification RR-G1602B (1984). The testing procedure consisted of first preparing sole samples (which were mounted upon a flat steel holder) to correspond to particular contaminant conditions. The samples were then placed upon the surface material and loaded with a $104 \mathrm{lb}$ vertical weight. This loading level corresponded closely to the average vertical loading exerted by a person of average weight while moving before a slip (Strandberg, 1983) and the sample was approximately the size of the toe area of an average male's shoe. Approximately $10 \mathrm{~s}$ after the weight was placed on the sample, a pulling force was gradually applied to the holder in a direction either lateral or longitudinal to the intended orientation of the slip resistant material. The pulling force was continuously recorded as it increased. At the point where movement began, the pulling force was ceased and the peak level was recorded.

The dependent variable was a static measure of the available surface slip resistance coefficient (ASRC), or simply the measured ratio of horizontal to vertical force at which movement occurs. The static ASRC appears to be a valid measure of slip potential, since at the start of a slip the velocity of the foot with respect to the floor surface is typically zero (Strandberg, 1983). The independent variables (Table 7) comprised seven different surfaces (six of which were described as being slip resistant by their manufacturers), four different sole materials, two different contaminants as well as no contaminants, and two orthogonal directions of the applied force (in relation to the normal orientation of the surface material). Five replicate measures were taken for each experimental condition.

\section{TABLE 7}

Independent variables considered during the experimental evaluation of the available slip resistance coefficient (ASRC)

\begin{tabular}{llll}
\hline Surface-type & Sole material & Contaminants & Force direction \\
\hline Slip resistant No. 1 & Crepe & None & Lateral \\
Slip resistant No. 2 & Leather & Water & Longitudinal \\
Slip resistant No. 3 & Ribbed-rubber & Diesel & \\
Slip resistant No. 4 & Oil-resistant-rubber & & \\
Slip resistent No. 5 & & & \\
Slip resistant No. 6 & & & \\
Smooth painted No. 7 & & & \\
\hline
\end{tabular}


Completion of the experiment resulted in table of 168 means. This factorial set of data described a large set of different walking or climbing scenarios.

\section{Statistical knowledge extraction}

Simply developing a set of 168 rules, where each rule defined the mean ASRC for a combination of experimental conditions was one feasible approach to system development. An alternative approach was to extract the knowledge imbedded in this data base by statistical methods. Such an approach would theoretically result in the development of meaningful rules rather than a large set of redundant data.

To evaluate the feasibility of the latter approach, we first used analysis of variance to uncover all significant main effects and interactions. The potential of predicting the ASRC was supported by the ANOVA, since the nonsignificant effects and error term together only corresponded to $6 \%$ of the variance. To predict the ASRC the significant main effects and interactions were then recoded as indicator variables (Chatterjee and Price, 1977) during regression analysis. It should be emphasized that the indicator variables were developed by recoding orthogonal variables measured in a complete factorial experiment. Consequently, the predictive weights of each indicator variable specified by the finally developed equation (Table 8) are meaningful, which is not generally the case in multiple regression analysis.

The regression equation explained $89 \%$ of the variance in the obtained values for the ASRC. Each term was highly significant with a small standard error and the variance/covariance matrix did not indicate problems with multicollinearity. Since the equation accurately predicted the ASRC, a decision was made to use the knowledge imbedded within the equation rather than the entire set of means.

TABLE 8

Summary of the regression equation used to predict the available slip resistance coefficient (ASRC)

\begin{tabular}{|c|c|c|c|c|c|}
\hline Factor & $\begin{array}{l}\text { Potent. } \\
\text { values }\end{array}$ & Weight & $\begin{array}{l}\text { Std. } \\
\text { error }\end{array}$ & T-Stat & Signif. \\
\hline 1. CONSTANT & (1) & 0.74 & 0.019 & 38.6 & 0.0000 \\
\hline 2. LEATHER-SOLE & $(0,1)$ & -0.25 & 0.022 & -11.7 & 0.0000 \\
\hline 3. CREPE-SOLE & $(0,1)$ & -0.15 & 0.021 & -7.2 & 0.0000 \\
\hline 4. OIL-RESISTANT-SOLE & $(0,1)$ & 0.17 & 0.018 & 9.1 & 0.0000 \\
\hline 5. WET $\times$ CREPE-SOLE & $(0,1)$ & -0.17 & 0.028 & -6.0 & 0.0000 \\
\hline 6. DIESEL $\times$ RUBBER-SOLE & $(0,1)$ & -0.29 & 0.017 & -17.4 & 0.0000 \\
\hline 7. STEP-RINGS & $(0,1)$ & 0.10 & 0.016 & 6.6 & 0.0000 \\
\hline 8. STEP-POINTS & $(0,1)$ & 0.06 & 0.020 & 3.0 & 0.0030 \\
\hline \multicolumn{6}{|l|}{ 9. PAINTED-STEP $\times$} \\
\hline RUBBER-SOLE & $(0,1)$ & -0.16 & 0.017 & -9.3 & 0.0000 \\
\hline \multicolumn{6}{|l|}{ 10. POINTS (NO EDGES)-STEP $\times$} \\
\hline CREPE or LEATHER-SOLE & $(0,1)$ & 0.13 & 0.036 & 3.7 & 0.0003 \\
\hline \multicolumn{6}{|l|}{ 11. SMOOTH-STEP $\times$} \\
\hline $\begin{array}{l}\text { LEATHER-SOLE } \times \\
\text { (NOT WET/WATER) }\end{array}$ & $(0,1)$ & -0.11 & 0.029 & -3.7 & 0.0003 \\
\hline \multicolumn{6}{|l|}{ 12. FORCE-DIR $\times$} \\
\hline STEP-EDGE-DIRECTION & $(-1,0,1)$ & 0.04 & 0.014 & 2.5 & 0.0123 \\
\hline \multicolumn{6}{|l|}{ 13. FORCE-DIR $\times$} \\
\hline STEP-EDGE-DIRECTION & $(-1,0,1)$ & 0.07 & 0.028 & 2.6 & 0.0101 \\
\hline \multicolumn{6}{|l|}{ STEP-EDGE-DIRECTION $\times$} \\
\hline HARD-RUBBER-SOLE & $(-1,0,1)$ & 0.11 & 0.028 & 3.9 & 0.0002 \\
\hline \multicolumn{6}{|l|}{ 15. FORCE-DIR $\times$} \\
\hline $\begin{array}{l}\text { STEP-RING-DIRECTION } \times \\
\text { LEATHER or RIBBED-SOLE }\end{array}$ & $(-1,0,1)$ & 0.07 & 0.016 & 4.5 & 0.0000 \\
\hline MULTIPLE $\mathrm{R}=0.94$ & RSQUARI & & S.E. $=$ & & \\
\hline
\end{tabular}




\section{Classification}

The meaning imbedded within the regression equation was difficult to discern because of the equation's complexity. It turns out, however, that the terms in the regression equation (Table 8) can be classified into three meaningful categories: simple sole material factors (terms 2, 3, and 4), sole-surface interlocking factors $(7,8,10,12,13$, 14 , and 15), and sole-surface stick factors $(5,6,9$, and 11). Each of these categories are conceptually independent. Therefore, given appropriate weighting, the contribution of factors not considered in the original experiment could be included in certain less quantitative forms of analysis. For example, interlocking effects due to other types of surface protrusions could be heuristically estimated instead of using the provided equations.

All three categories can also be further broken down into meaningful and conceptually useful subcategories. Sole-surface interlocking factors were subdivided into sole-point interlocking ( 8 and 10), sole-ring interlocking (7 and 15), and sole-edge interlocking $(12,13$, and 14). Sole-surface stick factors were subdivided into rubber-surface interaction factors $(5,6$, and 9) and leather-surface interaction factors (11). Simple sole material factors were subdivided into single elements.

\section{Rule development}

A set of 31 rules was developed, partitioned according to the meaningful categories and subcategories described above. Table 9 presents three illustrative rules. RULE134 organizes the three categories of factors described above. The ASRC is simply a constant plus the sum of the SOLESURFACE-INTERLOCKING-FACTOR, SIMPLE-SOLE-MATERIAL-FACTOR, and SOLESURFACE-STICK-FACTOR. Each of these factors must, of course, be determined before the ASRC can be determined. RULE130 shows how the SOLE-SURFACE-INTERLOCKING-FACTOR is calculated for one particular set of data. A total of 12 rules fell into this category. RULE140 shows how the RUBBER-SURFACE-INTERACTION-FACTOR is calculated for one combination of materials, contaminants and surface types. A total of 7 rules fell into this category. The remaining rules and factors were organized analogously.

\section{Incorporation of the rules into GSA}

Extending GSA required only minor extensions of its underlying model, since slips and falls naturally fell within the apply impact subproblem. For the generic portion of the system, additional rules

TABLE 9

Example rules estimating the ASRC

RULE134

If: (1) SOLE-SURFACE-INTERLOCKING-FACTOR is traced,

(2) SIMPLE-SOLE-MATERIAL-FACTOR is traced, and

(3) SOLE-SURFACE-STICK-FACTOR is traced

Then:(1) ASRC = SOLE-SURFACE-INTERLOCKING-FACTOR + SIMPLE-SOLE-MATERIAL-FACTOR + SOLE-SURFACESTICK-FACTOR + $0.74(1.0)$

\section{RULE130}

If: (1) the material the involved person's shoe soles are composed of is LEATHER,

(2) a characteristic describing the type of walking surface is PROTRUDING-RINGS, and

(3) one edge of the protruding rings is not higher than the other

Then: it is definite (1.0) that the calculated value of the SOLE-SURFACE-INTERLOCKING-FACTOR is 0.1

Source: Miller, Lehto, and Rhodes (1987).

\section{RULE140}

If: (1) the material the involved person's shoe soles are composed of is RUBBER

(2) the contaminant present on the walking surface is DIESEL, and

(3) a characteristic describing the type of walking surface is PAINTED

Then: it is definite (1.0) that the calculated value of the RUBBER-SURFACE-INTERACTION-FACTOR is -0.45 Source: Miller, Lehto, and Rhodes (1987). 
were added that related two new product functions of climbing and walking to the new specific danger cause of "required antislip force greater than available antislip force". Other very minor changes were also made.

Since the human's weight conceptually cancels out during the comparison of required and available antislip force (the ratio of the two forces depends only on the slip coefficients), GSA performs the product specific diagnosis by comparing the required and available slip coefficients. The ASRC is estimated using the 31 rules described above, while the required slip coefficient is implemented as a parameter that can be inputted by the user. The default value is currently the conservative value of 0.5 recommended in the OSHA 1910 standard and other sources. An obvious extension at this stage would be to determine the required value, using force platform data, for various tasks.

\section{SYSTEM IMPLEMENTATION}

\section{Example scenario}

A fall resulting in injury has taken place in an industrial setting. The safety practitioner is concerned with determining whether the walking surface upon which the fall took place is unsafe.
The practitioner knows or has access to information describing the surface, the type of shoe worn, and contaminants.

\section{Example session with GSA}

An interaction with GSA for the above scenario is presented in Table 10. GSA first asks for the name of the product and quickly produces generic outputs. From among these outputs, the specific cause of danger (the required antislip force is greater than the available antislip force) becomes the hypothesis the system seeks to confirm in the product specific diagnosis.

\section{The product specific diagnosis}

During the diagnosis, questions 4 and 5 are asked because the system needs to determine the required antislip coefficient. Analogously, questions 6 through 12 are used in determining the ASRC. Of interest is the provision of two different answers with certainty less than 1 to question 11 . Consequently, two possible values are calculated for the ASRC.

The system is unable to find any special safety problems, because the calculated available slip coefficients are both greater than 0.5. At this point, the product specific diagnosis is completed. Of interest is that the system efficiently developed the conclusion with a minimal set of questions.

TABLE 10

An example session with GSA.

(1) What is the name of the PRODUCT-1 that you wish to analyse?

** SLIP-RESISTANT-SURFACE

(2) Is analysis of impact related hazards for the slip-resistant surface desired?

** YES

The generic hazards associated with applying impact for the slip-resistant surface have been determined.

The generic hazards for the slip-resistant surface are as follows:

FALLING-HUMAN'S-KINETIC-ENERGY

FALLING-HUMAN'S-POTENTIAL-ENERGY

The generic dangers for the slip-resistant surface are as follows: FALLING-HUMAN-CONTACTS-SLIP-RESISTANT-SURFACE FALLING-HUMAN-CONTACTS-ENVIRONMENTAL-OBJECT

Generic causes of danger for the slip-resistance surface are as follows: IMPORTANT-CONNECTIONS-ARE-BROKEN

Specific causes of danger are as follows: REQUIRED-ANTISLIP-FORCE-GT-AVAILABLE-ANTISLIP-FORCE

Generic countermeasures are as follows: REDUCE-MOVING-HUMAN'S-KINETIC-ENERGY REDUCE-REQUIRED-ANTISLIP-FORCE INCREASE-AVAILABLE-ANTISLIP-FORCE 
TABLE 10 (continued)

(3) Do you wish to obtain a detailed diagnosis for the slip-resistant surface?

** YES

(4) Do you desire to input a value for the required antislip coefficient of the slip-resistant surface?

** RETURN INFO

The required antislip coefficient is needed in order to determine the safety of the surface. If you do not know what the value should be used for the required antislip coefficient, I will help you determine one.

** NO

(5) Does the OSHA 1910 standard apply to the environment the slip-resistant surface is used within?

** YES

I have determined that a reasonable value for the required antislip coefficient is 0.5 .

(6) How do you desire to describe the slip-resistant surface?

**?

one of: GENERICALLY

BY-MANUFACTURER

** BY-MANUFACTURER

(7) Give the manufacturer's name.

**?

one of: GRIP-STRUT

MORTION

NONE-OF-THESE

* * NONE-OF-THESE

(8) Can the characteristics describing the type of slip-resistant surface be best described as SERRADED EDGES?

** NO

(9) Can the characteristics describing the type of slip-resistant surface be best described as PROTRUDING-RINGS?

** YES

(10) Is one edge of the protruding rings higher than the other?

** YES

(11) When walking or climbing on the surface, is the horizontal force at the interface between the shoe and the slip-resistant surface normally exerted in the direction going from the higher edge of the ring to the lower edge of the ring?

* * YES (5) NO (5)

(12) What is the material the expected user's shoe soles are composed of?

**?

one of: LEATHER

RUBBER

CREPE

** LEATHER

The calculated values of the ASRC are as follows:

$0.66(0.5)$

$0.59(0.5)$

I was enable to determine any special apply-impact related safety problems for the slip-resistant surface.

(13) Is ELECTRICAL one of the foreseeable types of energy from the environment that may contact the slip-resistant surface?

** NO

Analysis of electrical energy related hazards is not relevant for the slip-resistant surface.

The generic hazards are analysed.

(14) Is analysis of the slip-resistant surface's compliance with standards desired?

** YES

Safety related standards for the product are as follows:

OSHA 1910

Federal Specification RR-G1602B

(15) List the standards which you wish to analyse for the slip-resistant surface.

** NONE

The safety analysis is as follows: DONE. 


\section{Other analysis}

The system then turns to other general safety problems defined by the generic model underlying GSA. The analysis of electrical energy related safety problems was quickly terminated, because the user's answer to question 13 allowed GSA to determine that such analysis was irrelevant for the product. Thermal and toxic material analysis were screened out because of high level rules associated with the product type.

In regard to safety standard's compliance GSA provides two relevant safety standards and then concludes the session.

\section{Discussion}

Many aspects of the interaction displayed in Table 10 have already been described. What should be emphasized is that the underlying model efficiently guided the problem solving process in a user friendly way. Additionally, the complexity of the underlying empirical knowledge became of little concern because the rules are designed so that it can not be applied inappropriately.

Placing the data into GSA also makes it available during the analysis of other safety problems. For example, in prying tasks the force exerted by the human could conceivably cause a slip. The system currently ignores this problem, but could be easily extended along these lines. This latter point describes an important advantage of the rule-based program as opposed to a traditional program.

Although a traditional user friendly program encoding a regression equation could easily be written, it would be difficult to quickly develop a large flexible problem solving system which flexibly applies the knowledge imbedded within the equation. Extending and/or modifying the traditional program (to accomodate changes in current knowledge, for example), would typically be difficult because the procedures which use the knowledge would also have to be modified. Of even greater difficulty in developing the traditional program would be the quick implementation of sophisticated user-system interaction, such as the explanation capability discussed earlier.

\section{CONCLUSIONS}

Of fundamental interest was that GSA efficiently incorporated new knowledge gathered using traditional experimental approaches. It can therefore be concluded, for certain problems in safety and ergonomics, that knowledge bases suitable for application by an expert systems can be developed using traditional methodologies. The results also demonstrate that well structured knowledge bases, as exemplified by the model underlying GSA, are maintainable.

The results also support the use of expert systems as a user friendly way of increasing access to both general and specialized knowledge.

As a final note, there are many easily implemented ways of further extending GSA. A general extension of this type would be to provide product specific recommendations along with the diagnosis. More detailed extensions include adding subproblems to those considered within interface biomechanics and increasing the empirical knowledge base, as in incorporating the ability to predict the required surface slip resistance values on the basis of force platform studies, and so on. The same can be said for safety problems involving the transfer of other forms of energy and for evaluating standards compliance. Such extensions are now being considered.

\section{REFERENCES}

Askren, W., 1985. New role for human factors in equipment design. Hum. Factors Bull., 28(4): 1-2.

Chatterjee, S. and Price, B., 1977. Regression Analysis by Example. Wiley, New York.

Coury, B., 1983. A specification for an ergonomic expert system. Department of Production Engineering and Production Management. University of Nottingham, unpublished report.

Chaffin, D., Herrin, G., Keyserling, W. and Garg, A., 1977. A method for evaluating the biomechanical stresses resulting from manual material handling jobs. Amer. Ind. Hyg. Assoc. J., 38(12): 662-675.

Driessen, G., 1970. Cause tree analysis: measuring how accidents happen and the probabilities of their causes. Paper presented at the 78th Annual Convention of the Americcan Psychological Association, Miami Beach, FL.

Drury, C. and Brill, M., 1983. Human factors in consumer product accident investigation. Hum. Factors, 25(3): $329-342$ 
Federal Specification RR-G-1602B, 1984. Federal specification: grating, metal, other than bar type. U.S. Government Printing Office, Washington, DC.

Haddon, W., 1973. Energy damage and the ten countermeasure strategies. J. Trauma, 13(4): 321-331.

Johnson, W.G., 1975. The management oversight and risk tree. J. Saf. Res., 7(1): 4-15.

Johnson, W.G., 1980. MORT Safety Assurance Systems. Marcel Dekker.

Lehto, M., Clark, D. and Miller, J., 1984. An isomorphism between fault tree analysis and LISP data structures. In: G. Salvendy (Ed.), Human-Computer Interaction. Elsevier, Amsterdam, pp. 297-300.

Lehto, M., 1985. An experimental application of knowledge engineering in the product safety domain. Ph.D. Dissertation, University of Michigan.

Lehto, M.R. and Miller, J.M., 1986. Warnings: Volume 1: Fundamentals, Design, and Evaluation Methodologies. Fuller Technical Publications, Ann Arbor, MI.

Martins, G., 1984. The overselling of expert systems. Datamation, 76-80.

Mital, A., 1983. Generalized model structure for evaluating/designing manual material handling jobs. Int. J. Prod. Res., 21(3): 401-412.

Miller, J.M., Lehto, M.R. and Rhodes, T., 1987. Predicting slip resistance in climbing systems. University of Michigan, unpublished Technical Report.
NIOSH, 1980. A work practices guide for manual lifting. U.S Department of Health Education and Welfare.

Ramsey, J.D., 1976. Ergonomic support of consumer product safety. Presented at the American Industrial Hygiene Conference.

Shortliffe, E.H. and Buchanan, B.G., 1984. A model of inexact reasoning in medicine. In: B.G. Buchanan and E.H. Shortliffe (Eds.), Rule-Based Expert Systems: The Mycin Experiments of the Standford Heuristic Programming Project, Addison-Wesley, Reading, MA, pp. 233-262.

Simon, H., 1969. The Sciences of the Artificial. MIT Press, Cambridge, MA, 2nd edn.

Snook, S.H., 1978. The design of manual handling tasks. Ergonomics, 21(12): 963-985.

Stefik, M., Aikins, J., Balzer, R., Benoit, J., Birnbaum, L. and Hayes-Roth, F., 1982. The organization of expert systems. Artif. Intell., 16(2): 141-170.

Strandberg, L., 1983. On accident analysis and slip resistance measurement. Ergonomics, 26(1).

Surry, J., 1968. Industrial accident research, a human engineering appraisal. Labour Safety Council, Toronto, Ontario.

Taylor, D.H., 1976. Accidents, risks and models of explanation. Hum. Factors, 18(4): 371-380.

Van Melle, W., 1980. The EMYCIN manual. Heuristic Programming Project, Stanford University, HPP-81-16. 